\title{
miR-200b downregulates CFTR during hypoxia in human lung epithelial cells
}

Sylwia Bartoszewska', Wojciech Kamysz¹, Bogdan Jakiela², Marek Sanak², Jarosław Króliczewski ${ }^{3}$ Zsuzsa Bebok ${ }^{4}$, Rafal Bartoszewski ${ }^{5^{*+}}$ (D) and James F. Collawn ${ }^{4+}$

\begin{tabular}{l}
\hline *Correspondence: \\
rafalbar@gumed.edu.pl \\
This article was specially invited by \\
the editors and represents work by \\
leading researchers. \\
${ }^{\dagger}$ Equal contributors \\
${ }^{5}$ Department of Biology and \\
Pharmaceutical Botany, Medical \\
University of Gdansk, Hallera 107, \\
$80-416$ Gdansk, Poland \\
Full list of author information is \\
available at the end of the article
\end{tabular}

* Correspondence: rafalbar@gumed.edu.pl the editors and represents work by leading researchers. ${ }^{\dagger}$ Equal contributors Department of Biology and Pharmaceutical Botany, Medical 80-416 Gdansk, Poland available at the end of the article

\begin{abstract}
Background: Hypoxic conditions induce the expression of hypoxia-inducible factors (HIFs) that allow cells to adapt to the changing conditions and alter the expression of a number of genes including the cystic fibrosis transmembrane conductance regulator (CFTR). CFTR is a low abundance mRNA in airway epithelial cells even during normoxic conditions, but during hypoxia its mRNA expression decreases even further.
\end{abstract}

Methods: In the current studies, we examined the kinetics of hypoxia-induced changes in CFTR mRNA and protein levels in two human airway epithelial cell lines, Calu-3 and 16HBE140-, and in normal primary bronchial epithelial cells. Our goal was to examine the posttranscriptional modifications that affected CFTR expression during hypoxia. We utilized in silico predictive protocols to establish potential miRNAs that could potentially regulate CFTR message stability and identified miR200b as a candidate molecule.

Results: Analysis of each of the epithelial cell types during prolonged hypoxia revealed that CFTR expression decreased after $12 \mathrm{~h}$ during a time when miR-200b was continuously upregulated. Furthermore, manipulation of the miRNA levels during normoxia and hypoxia using miR-200b mimics and antagomirs decreased and increased CFTR mRNA levels, respectively, and thus established that miR-200b downregulates CFTR message levels during hypoxic conditions.

Conclusion: The data suggest that miR-200b may be a suitable target for modulating CFTR levels in vivo.

Keywords: CFTR, HIF-1, micro-RNA 200b, hsa-miR-200b-3p

\section{Biomed Central}

\section{Background}

Cystic fibrosis is a lethal monogenic disease caused by mutations in the cystic fibrosis transmembrane conductance regulator (CFTR) [1]. The CFTR protein is a chloridebicarbonate channel that is expressed at low levels in epithelial cells of the airway, and at higher levels in epithelial cells in the intestine, pancreatic duct and male genital duct [2]. The post-transcriptional regulation of CFTR expression is controlled, at least in part, by microRNAs and this type of regulation has been demonstrated in Caco-2 cells, a human colon carcinoma cell line [3]. Studies by Gillen et al. [3] show that five microRNAs repress endogenous CFTR expression in this cell line, supporting the hypothesis

(c) The Author(s). 2017 Open Access This article is distributed under the terms of the Creative Commons Attribution 4.0 International License (http://creativecommons.org/licenses/by/4.0/), which permits unrestricted use, distribution, and reproduction in any medium, provided you give appropriate credit to the original author(s) and the source, provide a link to the Creative Commons license, and indicate if changes were made. The Creative Commons Public Domain Dedication waiver (http://creativecommons.org/ publicdomain/zero/1.0/) applies to the data made available in this article, unless otherwise stated. 
that differences in the miRNA profiles in various tissues modulate the expression of CFTR to different degrees.

In a transcriptomic mRNA and miRNA array-based analysis of the human colonic epithelial cell line HT29, Guimbellot and colleagues demonstrated that hypoxia mimetics treatment decreased CFTR message levels and that a number of miRNAs were upregulated [4]. Other studies have shown that miRNAs play a role in the posttranscriptional regulation of CFTR expression for both the wild-type protein and the most common mutation in cystic fibrosis, $\triangle$ F508 CFTR [5]. miRNAs are endogenous singlestranded RNAs that regulate the expression of specific genes at the posttranscriptional level $[6,7]$. They regulate gene expression by binding to a specific sequence in the 3' UTR or sometimes 5 'UTR of a target mRNA $[8,9]$.

Previous studies have shown that some miRNAs are induced during hypoxia and play a critical role in the cellular adaptive response to low oxygen levels [10]. Using in silico analysis (miRANDA and TargetScan algorithms) of miRNAs induced during hypoxia, we identified miR-200b as a potential novel regulator of CFTR mRNA levels. Experimental validation was confirmed in two human epithelial cell lines and in human primary lung epithelial cells and the results indicate that during hypoxia, miR-200b decreases CFTR mRNA levels in a time-course dependent manner.

\section{Methods}

\section{Cell lines and culture conditions}

Calu3 (ATCC ${ }^{\ominus}$ HTB-55 $^{\mathrm{mm}}$ ) and HEK293 (ATCC ${ }^{\bullet}$ CRL-1573) cells were obtained from ATCC. 16HBE14o- cells and HeLaWT were obtained as previously described [11, 12]. Cells were cultured in Minimum Essential modified Eagle's medium (Invitrogen) with $10 \%$ fetal bovine serum in a humidified incubator at $37{ }^{\circ} \mathrm{C}$ in $5 \% \mathrm{CO}_{2}$ in 6-well plates and allowed to grow to $70-80 \%$ confluence prior to the start of the experiments.

Primary human bronchial epithelial cells (NHBEC) were derived from brushings of bronchial mucosa obtained during bronchoscopy in normal individuals (i.e., patients referred for diagnostic bronchoscopy in which chronic airway disease was excluded during the further clinical investigation), and aged 30-64 (all donors were current nonsmokers). NHBEC were isolated by enzymatic digestion (pronase and DNAse I, SigmaAldrich, St. Louis, MO), cultured in supplemented bronchial epithelial growth medium (BEGM; Lonza, Basel, Switzerland) until confluent, and cryopreserved (passage 1) for further experiments. The sampling protocol was approved by Jagiellonian University Bioethics Committee, and informed consent was obtained from all participants. For experiments, thawed primary NHBEC were grown in BEGM medium (Lonza), as an adherent cell line, and maintained in culture until passage 5. Cells were seeded into 6well plates or $2 \mathrm{~cm}$ dishes and allowed to grow to $70-80 \%$ confluence prior to the start of the experiments.

\section{Induction of hypoxia}

Hypoxia was induced in a $\mathrm{CO}_{2} / \mathrm{O}_{2}$ incubator/chamber for hypoxia research (Invivo2, Baker Ruskin). Briefly, cells were cultured in $2 \mathrm{~cm}$ dishes at $0.9 \% \mathrm{O}_{2}$ for the time periods specified. Control cells were maintained in normoxic conditions in the same incubator and harvested at the specified times. 


\section{Isolation of RNA and microRNA}

Total RNA containing the microRNA fraction was isolated using the miRNeasy kit (Qiagen). RNA concentrations were calculated based on the absorbance at $260 \mathrm{~nm}$. RNA samples were stored at $-70{ }^{\circ} \mathrm{C}$ until use.

\section{5'UTR and 3'UTR CFTR Luciferase reporter assays}

A human 5'UTR CFTR promoter-driven firefly luciferase reporter construct (pCFTR-pLuc) was purchased from Panomics (Cat. \#: LR1020, Panomics Inc., Fremont, CA). This construct contains a 1000-bp fragment of the human CFTR $5^{\prime}$-UTR upstream of firefly luciferase as described in [13]. A human 3'UTR CFTR firefly luciferase reporter construct was purchased from GeneCopoeia (HmiT000948-MT06 - miRNA 3'UTR target expression clone for NM_000492.3) along with the control vector (CmiT000001-MT06 (miRNA Target clone control vector for pEZX-MT06).

To test the transcriptional and post-transcriptional activity of the human CFTR UTR regions, Calu3 cells and HEK293 were transfected with the constructs described above or with control plasmids provided by Panomics/GeneCopoeia. Twenty-four hours before experiments, cells were seeded onto 6-well plates at $\sim 40 \%$ confluency and transfected using Lipofectamine 2000 (Invitrogen). For the specified experiments, miR-200b and miR-200c analogs were cotransfected. Each well received $2 \mu \mathrm{g}$ of total plasmid DNA and $1 \mu \mathrm{g}$ of a vector of interest plus $1 \mu \mathrm{g}$ of Renilla luciferase as an internal control for the 5'UTR or 3'UTR constructs. For the 5'UTR and 3'UTR cotransfections ( $2 \mu \mathrm{g}$ total), $1 \mu \mathrm{g}$ of each reporter vector was used as well as $1 \mu \mathrm{g}$ of Renilla luciferase as an internal control. miR-200b mimic was used at final concentration of $10 \mathrm{nM}$. At the time points indicated, cells were lysed using luciferase assay lysis buffer (Promega) and firefly/Renilla luciferase activities were measured using the Dual-Luciferase Reporter Assay (Promega) according to the manufacturer's protocol. Results in treated cells were plotted as the percent decrease in arbitrary light units compared with control cells.

\section{Measurement of mRNA and miRNA levels using quantitative Real Time PCR (qRT-PCR)}

We used TaqManOne-Step RT-PCR Master MixReagents (Applied Biosystems) as described previously $[14,15]$ using the manufacturer's protocol. The relative expressions were calculated using the comparative relative standard curve method [16]. We used $18 S$ rRNA as the relative control for our studies. We also validated this relative control against another housekeeping gene, TATA-binding protein $(T B P)$. As relative controls for miRNA quantification, we validated and used RNU48. TaqMan probes ids used were: CFTR Hs00357011_m1; 18S - Hs99999901_s1; TBP - Hs4332659_m1; RNU48 - 001006; miR200b - 002251; miR-200c - 002300.

\section{miRNA analog transfections}

miR-200b mimic (id MC10492) and antagomiR (id MH10492), as well as miR-200c mimic (id MC11714) and antagomiR (id MH11714), were purchased from Ambion. Cells were transfected using the Lipofectamine RNAiMax according to manufacturer's protocol. miR-200b/c mimics and antagomiRs were used at final concentrations of $10 \mathrm{nM}$ and $20 \mathrm{nM}$, respectively. The transfected cells were cultured for 2 days prior to 
further analysis. The degree of miRNA over-expression or knockdown was determined by qRT-PCR (Additional file 1: Figure S1B). cel-miR-67 was used as a control (Ambion assay id MC22484). As additional controls, Ambion siRNA Negative Control 1 (no. 4390843), Ambion mimic control (no. 4464060) and Ambion antagomiR control (no. 4464076) were used as well.

\section{Western blots}

Cells were lysed in RIPA buffer $(150 \mathrm{mM} \mathrm{NaCl}, 1 \% \mathrm{NP}-40,0.5 \%$ sodium deoxycholate, $0.1 \%$ SDS, $50 \mathrm{mM}$ Tris- $\mathrm{HCl}$, pH 8.0) supplemented with protease Inhibitor Complete Mini (Roche) on ice for $15 \mathrm{~min}$. The cell lysates were rotated at $4{ }^{\circ} \mathrm{C}$ for $30 \mathrm{~min}$ and the insoluble material was removed by centrifugation at 15,000 g for $15 \mathrm{~min}$. Protein concentrations were determined by BioRad ${ }^{\mathrm{Tm}}$ Protein Assay using bovine serum albumin (BSA) as a standard. Following the normalization of protein concentrations, lysates were mixed with an equal volume of $2 \mathrm{X}$ Laemmli sample buffer and incubated for 5 min at $95{ }^{\circ} \mathrm{C}$ prior to separation by SDS PAGE on stain-free TGX gradient gels (BioRad). Following SDS-PAGE, the proteins were transferred to polyvinylidene fluoride membranes (300 $\mathrm{mA}$ for $90 \mathrm{~min}$ at $4{ }^{\circ} \mathrm{C}$ ). The membranes were then blocked with BSA (Sigma-Aldrich) dissolved in PBS/Tween-20 (3\% BSA, 0.5\% Tween-20 for 1-2 h), followed by immunoblotting with the primary antibody specified for each experiment CFTR (Merck MM13-4 diluted at 1:1000), HIF-1 $\alpha$ (Abcam ab16066, diluted at 1:1000); and beta ACTIN (Abcam ab1801, diluted at 1:1000). After the washing steps, the membranes were incubated with goat anti-rabbit IgG ( $\mathrm{H}+\mathrm{L}$ chains) or with goat anti-mouse IgG $(\mathrm{H}+\mathrm{L})$ HRP-conjugated secondary antibodies (BioRad) and detected using ECL (Amresco). Densitometry was performed using Image Lab software v. 4.1 (BioRad).

\section{Statistical analysis}

The results were expressed as the mean \pm standard deviation (SD). Statistical significance among means was determined using the Student's t-test (two samples, paired and unpaired) [17]. Analyzes were performed with Dell Statistica version 13 (Dell Inc., 2016).

\section{Results}

Downregulation of CFTR during hypoxia involves post-transcriptional HIF-1-dependent mechanisms

Previous studies reported that CFTR protein and mRNA levels were reduced during hypoxia in human lung epithelial cells $[18,19]$. However, these reports were limited to chemical hypoxia induction and a single time point of physiological hypoxia and therefore did not provide the information about the dynamics of hypoxic CFTR message downregulation. Hence, to examine the kinetics of hypoxia-induced changes of CFTR protein and mRNA levels, we performed time-course studies during physiological hypoxia in two lung epithelial cell lines, Calu3 cells (epithelial lung adenocarcinoma; derived from metastatic site: pleural effusion) and 16HBE14o- cells (SV40-immortalized human bronchial epithelial cells). Both Calu3 and 16HBE14o- cell lines are commonly used in vitro for studying CFTR biogenesis and function. As shown in Fig. 1a, the changes in CFTR mRNA profiles show a significant decrease in mRNA after $8 \mathrm{~h}$ in 

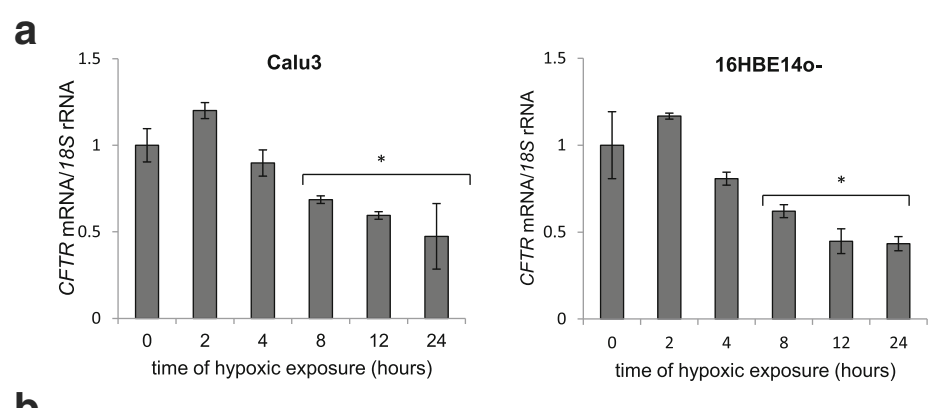

b

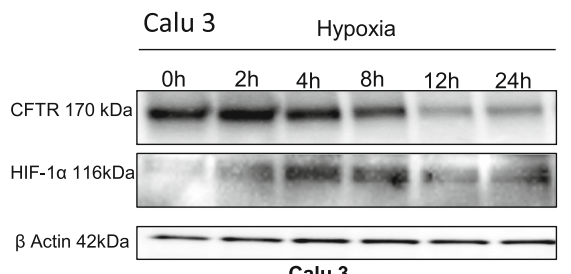

16HBE140- Hypoxia
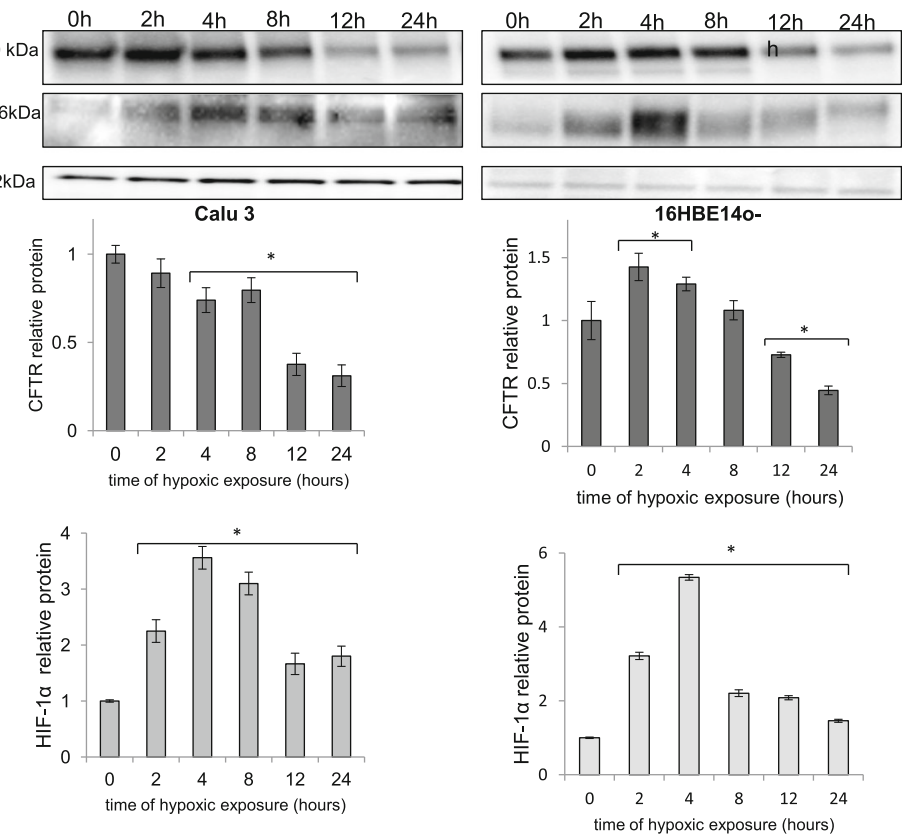

Fig. 1 Regulation of CFTR during hypoxia in human lung epithelial cells, Calu3 cells (left panels) and 16HBE140- cells (right panels). a CFTR mRNA is reduced during hypoxia. CFTR mRNA levels were monitored in qRT-PCR experiments. The results from 3 independent experiments $(n=12)$ are plotted normalized to $18 \mathrm{~S}$ rRNA levels and expressed as a fold-change over the normoxic control. b Hypoxia sequentially increases HIF-1a protein levels and reduces CFTR protein levels. Protein expression levels of were monitored with SDS-PAGE and Western Blot and normalized to $\beta$-actin levels. Two individual samples ( $4 \mu \mathrm{g}$ of total protein per lane) were tested for each time point and the experiments were repeated twice. Error bars represent standard deviations. Significant changes $(P<0.05)$ are marked with an asterisk

both cell lines and correlate well with the CFTR protein changes (Fig. 1b). During the early stages of hypoxia up to $8 \mathrm{~h}$, CFTR protein levels remain either mostly unchanged (Calu3 cells) or slightly induced (16HBE14o- cells), whereas, under chronic hypoxia (after $12 \mathrm{~h}$ ), CFTR levels are significantly reduced and drop below half of the normoxic levels in both cell lines.

In a previous study, Zheng and coworkers reported that HIF-1 is responsible for decreases in CFTR mRNA and protein [18], and therefore we followed the levels HIF1A message and HIF-1 $\alpha$ protein during the hypoxia time course as well (Fig. 1a, b). In both Calu3 and 16HBE14o- cells, the maximum of HIF-1 $\alpha$ protein increases during hypoxia immediately preceded the decline in CFTR protein and mRNA, which is consistent with previous studies in intestinal epithelia that HIF-1 decreases CFTR mRNA levels 

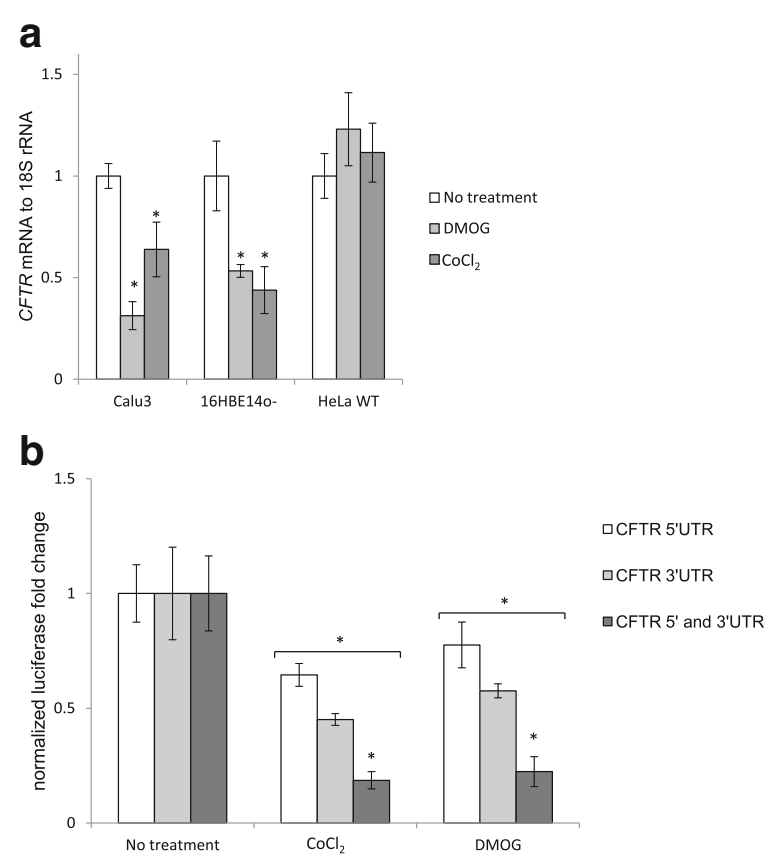

Fig. 2 Downregulation of CFTR expression during hypoxia is HIF-1 dependent and relies on both the $5^{\prime}$ and 3' UTRs of CFTR mRNA. a Calu3, 16HBE14o- and HeLa WT CFTR cells were treated with hypoxia mimetics $(500 \mu \mathrm{M}$ DMOG for $12 \mathrm{~h}$ (light grey) and $200 \mu \mathrm{M} \mathrm{CoCl}$ for $12 \mathrm{~h}$ (dark grey)) and the mRNA levels were monitored in qRT-PCR experiments. CFTR mRNA levels from 2 independent experiments $(n=8)$ are plotted relative to 185 rRNA levels and expressed as a fold change over the untreated control. b Calu3 cells were transfected with 5'UTR CFTR luciferase reporter (white), 3'UTR CFTR luciferase reporter (light grey) and cotransfected with both $5^{\prime} U T R$ and $3^{\prime} U T R R$ CFTR luciferase reporters (dark grey) and treated with hypoxia mimetics (500 $\mu \mathrm{M}$ DMOG or $200 \mu \mathrm{M} \mathrm{CoCl}$ for $12 \mathrm{~h}$ ) and the luciferase activity was monitored. These reporters were normalized to internal controls (Renilla) firefly luciferase activities from 2 independent experiments $(n=6)$ and plotted and expressed as a fold-change over non-treated control. Error bars represent standard deviations. Significant changes $(P<0.05)$ are marked with an asterisk

during hypoxia. To test this hypothesis using another approach, we utilized hypoxia mimetics $\left(\mathrm{CoCl}_{2}\right.$ and dimethyloxalylglycine (DMOG)) that stabilize the HIF-1 $\alpha$ protein and thus induce HIF-1 transcriptional activity [20]. We analyzed the related changes in CFTR mRNA in Calu3 and 16HBE14o- cells as well as in a HeLa cell line that expresses recombinant CFTR mRNA (HeLaWT) that does not contain the $5^{\prime}$ or $3^{\prime}$ UTRs of CFTR (Fig. 2a). The chemically stabilized HIF-1 activity mediated by the hypoxia mimetics decreased CFTR mRNA in both Calu3 and16HBE14o- cells, whereas it had no effect on exogenous CFTR mRNA levels in HeLaWT cells.

While these data confirm that during hypoxia, CFTR mRNA is decreased, they do not address whether this is a transcriptional and/or post-transcriptional HIF-1dependent mechanism. Since we did not observe a clear negative correlation between CFTR mRNA and HIF-1 $\alpha$ expression profiles (Fig. 1), this suggested the possibility that other HIF-1-dependent secondary post-transcriptional factors might be responsible for the decline in CFTR message and protein expression. To examine this hypothesis, we tested the function of the CFTR 5'UTR and 3'UTR mRNAs during hypoxia using specific luciferase reporters. As shown in Fig. 2b, the hypoxia mimetics $\mathrm{CoCl}_{2}$ and DMOG reduced both 5' UTR- and 3'UTR-dependent luciferase expression, suggesting that both untranslated regions of CFTR mRNA are involved in HIF-1-dependent reduction of CFTR mRNA. Interestingly, luciferase 
expression from the CFTR 3'UTR reporter construct was more inhibited than the 5 'UTR reporter, and that the two effects were additive. This suggested a synergistic effect of both UTRs in reducing CFTR mRNA during hypoxia and the involvement of both the 5'UTR and 3'UTR in transcriptional/post-transcriptional HIF-1dependent mechanisms.

\section{miR-200b is induced by hypoxia in a HIF-1-dependent manner in human lung} epithelial cells

The expression of many miRNAs has been shown to be HIF-1 dependent under hypoxia [21]. To test the hypothesis that such a HIF-1-dependent miRNA could contribute to CFTR downregulation, we analyzed the CFTR 3'UTR sequence for potential binding sites using the miRANDA and TargetScan algorithms [22, 23]. Using this approach, we

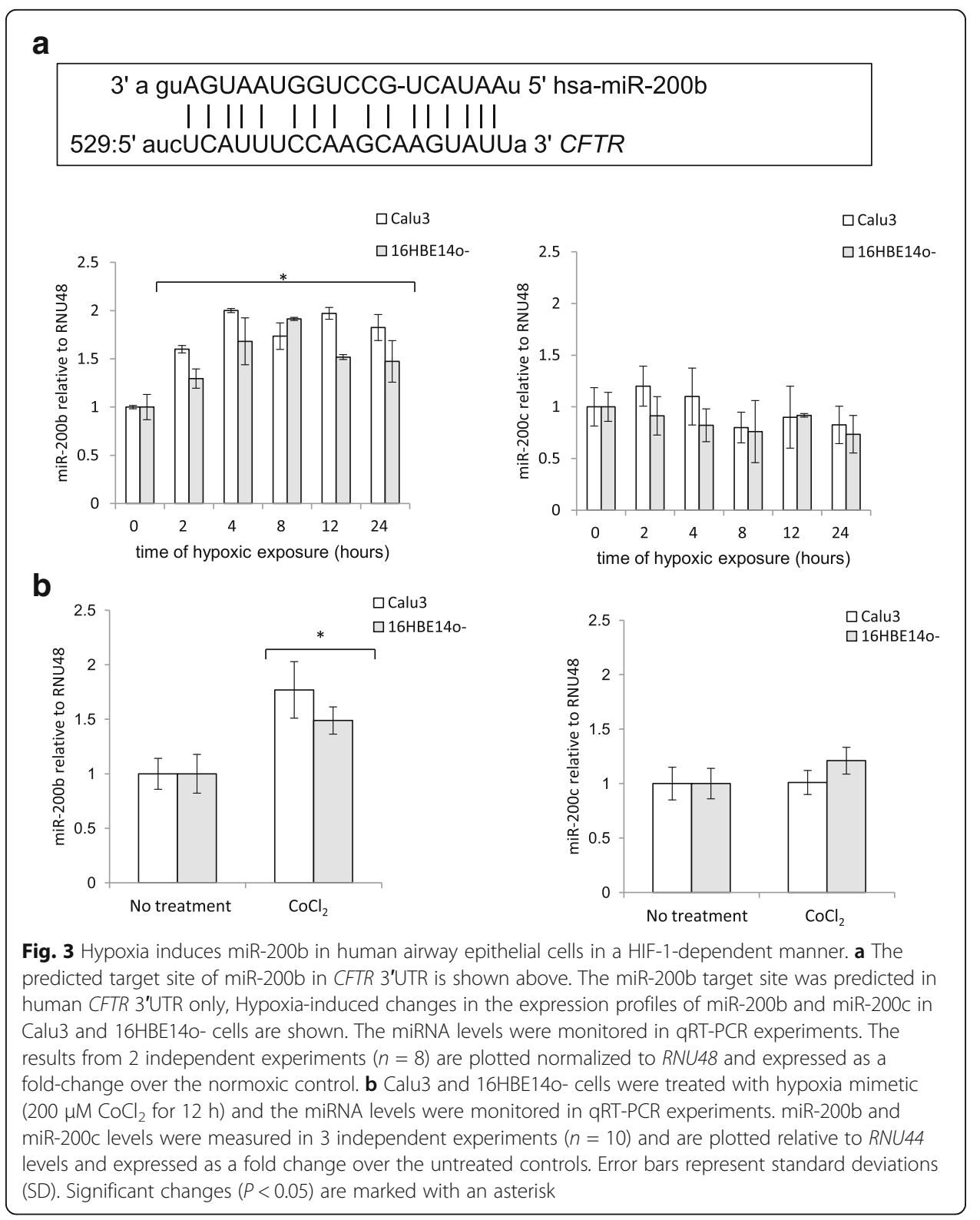


identified a potential target site for miR-200b/200c at position 529 bases from the stop codon in the 3'UTR of CFTR mRNA (Fig. 3). Since the expression of miR-200b and miR-200c was previously reported to be hypoxia-dependent in human endothelial cells, we tested their expression profiles during hypoxia in the Calu3 and 16HBE14o- cells. As shown in Fig. 3a, miR-200b was induced up to 2-fold during the hypoxia time course in both cell lines, whereas miR-200c was not elevated and therefore probably not involved in CFTR regulation during hypoxia. The miR-200b levels were elevated to a maximum level at $4 \mathrm{~h}$ and that correlated well with HIF-1's maximal expression, and importantly, miR-200b levels remained elevated throughout the 24-h test period. Furthermore, the increase in miR-200b levels correlated negatively with the respective decrease in CFTR mRNA and protein, and supported miR-200b's role in regulating CFTR expression.

Although in our previous studies in human primary endothelial cells (HUVECs), we identified a HIF response element (HRE) consensus in the proximity of miR-200b sequence, the hypoxia mimetics had limited impact on miR-200b's expression [24]. However, miRNA expression, as well as HIF-1 activity, is often tissue-specific, and therefore we tested whether the induction of HIF-1 activity would affect miR-200b expression in lung epithelial cells. As shown in Fig. 3b, $\mathrm{CoCl}_{2}$ induced HIF-1 activity and resulted in the elevation of miR-200b levels in both Calu3 and 16HBE14o- cells, suggesting that hypoxic induction of this miRNA is HIF-1 dependent. Furthermore, $\mathrm{CoCl}_{2}$ treatment had no significant effect on miR-200c expression.

\section{miR-200b binds to CFTR's 3'UTR}

Although the miRNAs recognize specific target sequences, these sequences (6-8 nt) can be present in the 3'UTRs of many different genes. Hence, in order to exclude indirect effects of miR-200b on CFTR expression, we utilized 3'UTR luciferase reporter. Briefly, a plasmid containing the 3' UTR of human CFTR gene was tested in a luciferase gene construct that was co-expressed in human embryonic kidney cells 293 (HEK293) in the presence and absence of a miR-200b analog (mimic). HEK293 cells were used since they express very low endogenous levels of miR-200b/c. As shown in Fig. 4a, miR-200b overexpression resulted in significantly reduced luciferase expression compared to the no treatment control. Furthermore, a similar experiment with miR-200c that has only one base difference in seed sequence from miR-200b did not result in a luciferase signal reduction (Fig. 4b), confirming the direct interaction between miR-200b and its target site at 3'UTR of CFTR mRNA.

Next, we tested the effects of miR-200b overexpression and inhibition on CFTR mRNA levels after $12 \mathrm{~h}$ of hypoxia. The miR-200b upregulation with mimic reduced CFTR mRNA in hypoxia and normoxia in both Calu3 and 16HBE14o- (Fig. 5a). Furthermore, the inhibition of miR-200b activity with antagomiR increased CFTR mRNA in both cell lines (Fig. 5a). In parallel, we followed miR-200b analogs effect on CFTR protein levels. As shown in Fig. 5b, in normoxia and during hypoxia, miR-200b overexpression resulted in the reduction of CFTR protein levels in 16HBE14o- cells. Whereas in Calu3 cells, mimic only had an effect during normoxia, although both cell lines had elevated CFTR protein with the antagomir treatment during hypoxia, confirming the physiological effect of miR-200b CFTR expression during low oxygen levels. 


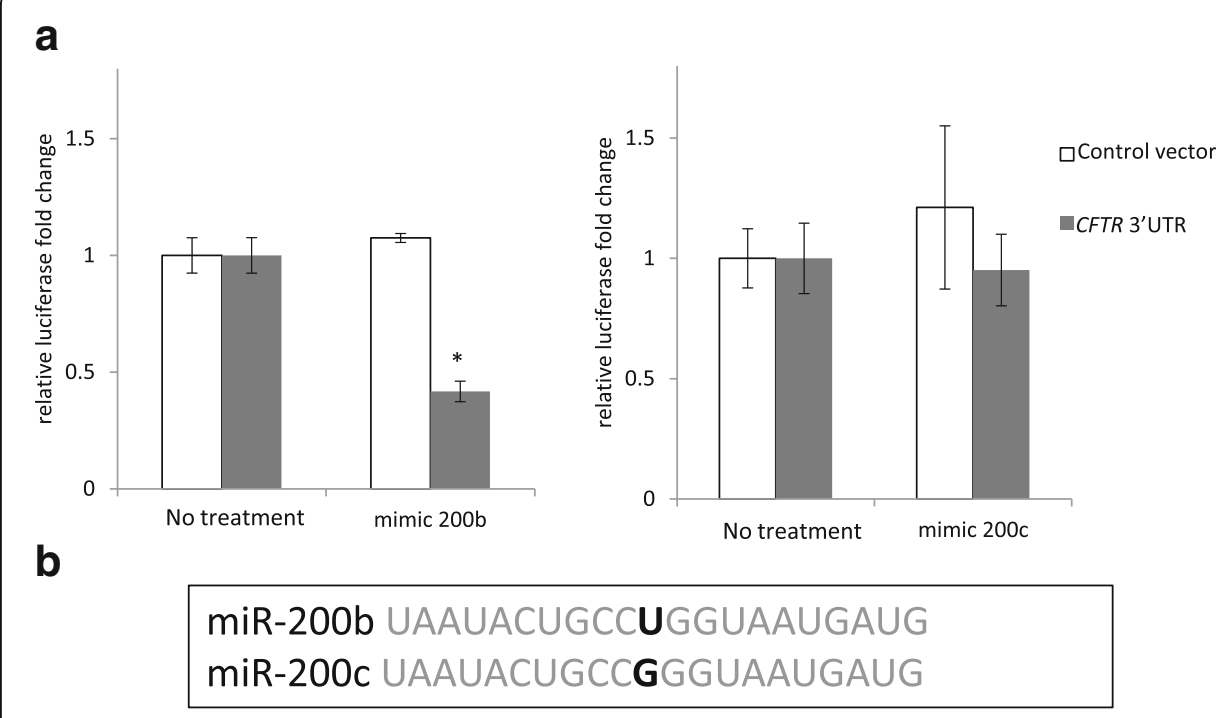

Fig. 4 miR-200b binds to the predicted target sequence in the CFTR 3'UTR. a HEK293 cells were transfected with 3'UTR CFTR luciferase reporter construct alone (white) or together with miR-200b mimic (grey, left panel) or miR-200c mimic (grey, right panel). Similar experiments were performed on control vector that did not contain the miR-200b/miR-200c target site (not shown). Data were normalized to control Renilla luciferase activities from 2 independent experiments $(n=6)$ and are expressed as a fold-change over control. Error bars represent standard deviations (SD). Significant changes $(P<0.05)$ are marked with an asterisk. $\mathbf{b}$ The comparison of miR-200b and miR-200c seed sequences is shown

Given that miRNA levels and function in cancer cell lines often differs from primary cells, we examined miR-200b's impact on CFTR expression in primary human lung cells (NHBEC Normal Human Bronchial Epithelial Cells) obtained from 3 donors. As shown in Fig. 6a, miR-200b was significantly induced $\sim 2.5$-fold during hypoxia in NHBECs, while the CFTR mRNA reduced by about $50 \%$ during normoxia $(P=0.07)$ and less so during hypoxia (Fig. 6b). However, the smaller effect during hypoxia may be due to already very low CFTR mRNA basal levels in NHBECs (approximately 50 fold lower than in immortalized cells, Additional file 1: Figure S1A). Inhibition of miR-200b by antagomiR, however, significantly increased CFTR mRNA levels in both conditions (Fig. 6b), confirming the physiological relevance of results obtained in immortalized cell lines. Importantly, we were able to observe a significance decrease in CFTR protein levels with miR-200b overexpression during normoxia, and a decrease during hypoxia, though it was not significant $(P=0.08)$ in NHBECs (Fig. 6c). miR-200b antagomiR treatment resulted in a significant increase in CFTR protein levels during both normoxia and hypoxia and this supported by the changes in CFTR mRNA as well. Importantly, our results obtained in primary human lung cells confirmed that miR-200b regulates CFTR expression.

\section{Discussion}

The regulation of CFTR expression appears to be tissue specific and understanding its regulation is important in potential therapies for cystic fibrosis (CF) given that many of the disease causing mutations result in lower expression of this critically vital gene. Transcriptional regulation of CFTR is complex and includes elements within the 


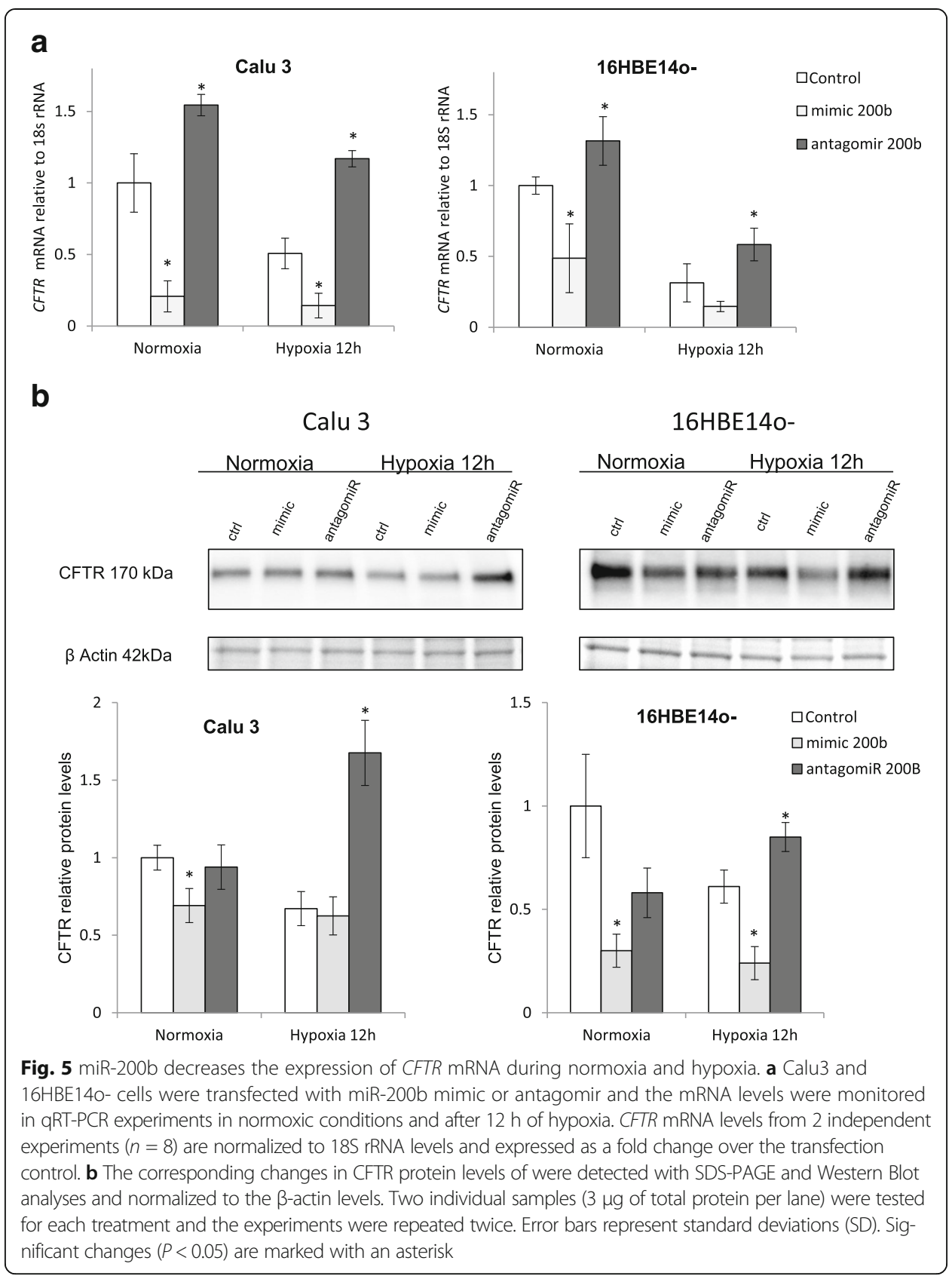

promoter and intronic enhancers (reviewed in [25]). It has also been established that miRNA networks regulate CFTR expression as well [5, 26].

Although the role of miRNAs in posttranscriptional gene regulation is clearly established, it is now becoming evident that recent studies have shown that specific alterations in miRNA expression occur in cystic fibrosis (reviewed in [27]). Moreover, differences in miRNA expression are also present in chronic obstructive pulmonary disease, asthma, lung inflammation, and in smoke exposure in humans [28], suggesting that miRNA network changes can potentially influence disease pathogenesis. For CF, this is illustrated for the $\triangle \mathrm{F} 508$ CFTR mutation, the most common mutation in CF, by studies that show that there is increased expression of miR-145, miR-223, and miR-494 

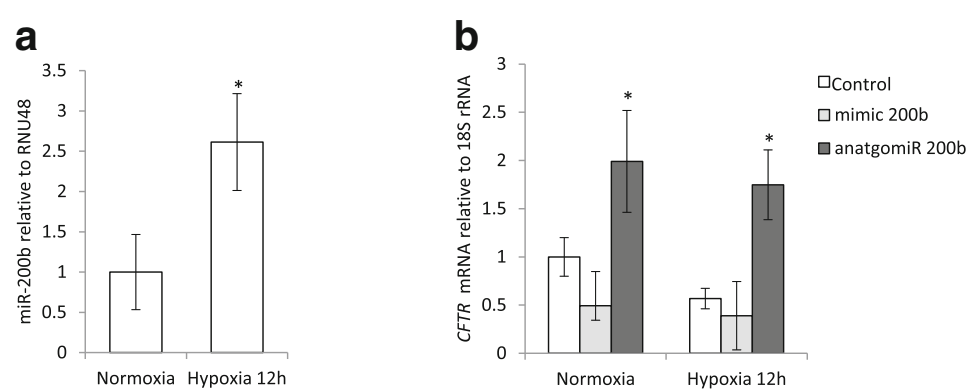

C

NHBEC
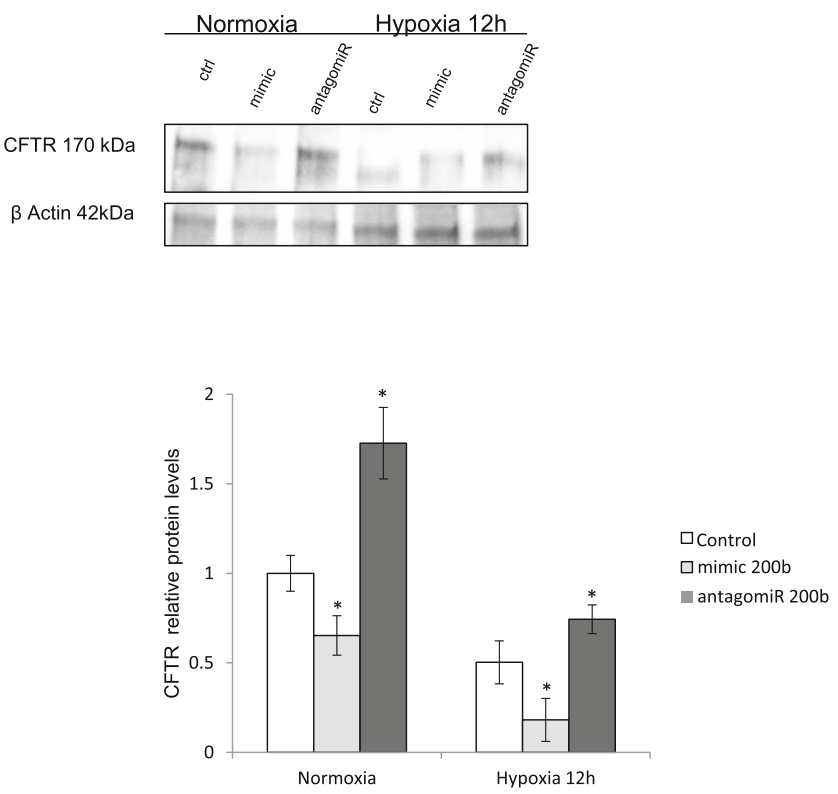

Fig. 6 miR-200b decreases the expression of CFTR mRNA during normoxia and hypoxia in primary normal human bronchial epithelial cells (NHBEC). a The levels of miR-200b after $12 \mathrm{~h}$ of hypoxia in NHBECs from 3 independent experiments $(n=6)$ are plotted normalized to RNU44 levels and expressed as a fold change over the normoxia control. b NHBEC cells were transfected with miR-200b mimic or antagomir and the mRNA levels were monitored in qRT-PCR experiments in normoxic conditions and after $12 \mathrm{~h}$ of hypoxia. CFTR mRNA levels from 2 independent experiments $(n=8)$ are plotted normalized to 185 rRNA levels and expressed as a fold change over the transfection control. c The corresponding changes of CFTR protein levels were monitored with SDS-PAGE and Western Blot analysis and normalized to the $\beta$-actin levels. Two individual samples ( $3 \mu \mathrm{g}$ of total protein per lane) were tested for each treatment and the experiments were repeated twice. Error bars represent standard deviations $(\mathrm{SD})$. Significant changes $(P<0.05)$ are marked with an asterisk

in vivo in the bronchial epithelium of $\triangle \mathrm{F} 508$ patients and this correlated with decreased CFTR expression [25, 29]. Furthermore, the introduction of a miRNA site through a mutation has been shown to increase the affinity of a miRNA that in vitro lowers the expression of the CFTR protein [30].

Using transcriptomic mRNA and miRNA-array-based experiments in colonic epithelial cells, Guimbellot and colleagues demonstrated that a number of genes were up- or down-regulated during hypoxia and CFTR was one of those genes that was downregulated [4]. It has also been reported that HIF-1 expression decreases CFTR expression in intestinal epithelium [18], suggesting that transcriptional regulation controls CFTR repression during hypoxia. The goal of the present study was to determine the relative contribution of miRNA-mediated post-transcriptional mechanisms as well. 
Our in silico predictions indicated that miR-200b and miR-200c were putative candidates for CFTR posttranscriptional regulation. Using a hypoxia time course, we show that CFTR mRNA expression decreased after $8 \mathrm{~h}$ in both human airway epithelial cell lines, whereas HIF- $1 \alpha$ protein expression was elevated as early as $2 \mathrm{~h}$. Interestingly, the CFTR protein levels were not dramatically lower until $12 \mathrm{~h}$, suggesting that more than just HIF-1 suppression of CFTR expression was occurring. To test for potential miRNA effects on CFTR expression, we utilized luciferase reporter constructs containing either the human CFTR 5'UTR, the 3'UTR or both and, in conjunction with chemical mimics of hypoxia that stabilized HIF-1 protein expression. We found that the 3'UTR was the more important region for suppression of expression, but that both regions were important and additive in their effects.

To differentiate between miR-200b and miR-200c, we found that miR-200b was elevated in both cell lines during hypoxia, whereas miR-200c was not. Furthermore, in Guimbellot et al.'s analysis of HT29 colonic epithelial cells, they identified 28 miRNAs that were upregulated during hypoxia, and miR-200b was one of them [4]. This suggested that miR-200b could have effects on CFTR mRNAs in more than just airway epithelia. Using the 3'UTR CFTR luciferase constructs we also demonstrated that miR-200b had a direct effect on luciferase expression, and this clearly established a direct effect on CFTR message levels. Final support for the role of miR-200b comes from the negative and positive effects of miR-200b mimics and antagomirs on CFTR expression changes, including the results in the primary airway cells. Taken together, the results suggest that during low oxygen conditions which could occur in various lung pathologies, miR-200b is upregulated and has a direct inhibitory effect on CFTR message and protein expression in human airway epithelial cells.

\section{Conclusions}

In summary, our studies suggest that the HIF-1 dependent physiological changes in miR-200b levels in human airway epithelia under hypoxia contribute directly to CFTR downregulation during hypoxia. Hence, this results complement previous studies

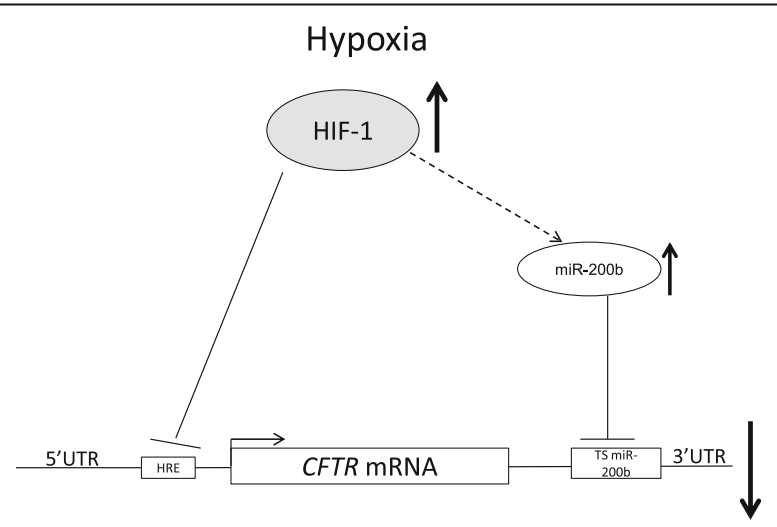

Fig. 7 Model for negative regulation of CFTR expression during hypoxia by HIF-1 and miR-200b. During hypoxia, HIF-1 activity is induced and HIF-1 binds to the hypoxia response element (HRE) sequence located in CFTR 5'UTR and has been reported to decrease CFTR expression (Zheng et al. [18]). Our studies show that HIF-1 induces miR-200b expression and binds to the target sequence (TS miR-200b) located at the $3^{\prime} U T R$ of CFTR mRNA, which further decreases CFTR mRNA and protein expression 
indicating HIF-1's direct transcriptional effects on downregulation of CFTR with an additive post-transcriptional mechanism that involves a hypoxia-induced miRNA (Fig. 7). Furthermore, stabilization of CFTR protein levels during hypoxia through inhibition of miR-200b's actions may provide a novel therapeutic opportunity for increasing CFTR expressions levels during various lung pathologies.

\section{Additional file}

Additional file 1: Figure S1. Endogenous levels of miR-200b in NHBEC, Calu3 and 16HBE140- cells. (A) Comparison of CFTR mRNA (white) and miR-200b (grey) relative levels between NHBEC (primary cells), Calu3 and 16HBE140- cells during normoxic conditions. CFTR mRNA levels from 2 independent experiments $(n=8)$ are plotted normalized to $18 \mathrm{~S}$ rRNA levels and expressed as a fold change over the NHBEC levels. miR-200b levels from 2 independent experiments $(n=8)$ are plotted normalized to RNU48 levels and expressed as a fold change over the NHBEC levels. (B) NHBEC, Calu3 and 16HBE140- cells were transfected with miR-200b antagomir (left) or mimic (right) and the miRNA levels were monitored in GRT-PCR experiments. miR-200b levels from 2 independent experiments $(n=8)$ are plotted normalized to RNU48 levels and expressed as a fold change over the transfection control. Error bars represent standard deviations (SD). Significant changes $(P<0.05)$ are marked with an asterisk. (PDF $348 \mathrm{~kb})$

\section{Abbreviations}

CFTR: Cystic fibrosis transmembrane conductance regulator; HIF: Hypoxia inducible factor; miRNA: microRNA

\section{Acknowledgments}

Not applicable.

\section{Funding}

This work has been supported by National Science Center "SONATA BIS" Program under contract UMO-2015/18/E/ NZ3/00687 (R.B.) and NIH P30 DK072482 (J.F.C.).

\section{Availability of data and materials}

The data sets generated and/or analyzed during this study are available from the corresponding author upon reasonable request.

\section{Authors' contributions}

Conceived and designed the experiments: SB RB. Performed the experiments: SB RB All authors analyzed the data. Contributed reagents/materials/analysis tools: RB ZB WK BJ MS. Wrote the paper: RB and JFC. All authors read and revised the final version of the manuscript. All authors read and approved the final manuscript.

\section{Ethics approval and consent to participate}

The NHBEC cells were obtained according to Jagiellonian University Medical College Bioethics Committee agreement $\mathrm{KBET} / 68 / \mathrm{B} / 2008$. The experiments with use of anonymous NHBEC cell lines were performed according to Jagiellonian University Medical College Bioethics Committee agreement KBET/284/B/2014.

\section{Consent for publication}

Not applicable.

\section{Competing interests}

The authors declare that they have no competing interests.

\section{Publisher's Note}

Springer Nature remains neutral with regard to jurisdictional claims in published maps and institutional affiliations.

\section{Author details}

'Department of Inorganic Chemistry, Medical University of Gdansk, Gdansk, Poland. 'Department of Medicine, Jagiellonian University Medical College, Krakow, Poland. ${ }^{3}$ Department of Chemical Biology, Faculty of Biotechnology, University of Wroclaw, Wroclaw, Poland. ${ }^{4}$ Department of Cell, Developmental and Integrative Biology, University of Alabama at Birmingham, Birmingham, USA. ${ }^{5}$ Department of Biology and Pharmaceutical Botany, Medical University of Gdansk, Hallera 107, 80-416 Gdansk, Poland.

Received: 14 September 2017 Accepted: 17 October 2017

Published online: 15 November 2017

\section{References}

1. Rowe SM, Miller S, Sorscher EJ. Cystic fibrosis. N Engl J Med. 2005:352:1992-2001.

2. Trapnell BC, Chu CS, Paakko PK, Banks TC, Yoshimura K, Ferrans VJ, Chernick MS, Crystal RG. Expression of the cystic fibrosis transmembrane conductance regulator gene in the respiratory tract of normal individuals and individuals with cystic fibrosis. Proc Natl Acad Sci U S A. 1991;88:6565-9. 
3. Gillen AE, Gosalia N, Leir SH, Harris A. MicroRNA regulation of expression of the cystic fibrosis transmembrane conductance regulator gene. Biochem J. 2011;438:25-32.

4. Guimbellot JS, Erickson SW, Mehta T, Wen H, Page GP, Sorscher EJ, Hong JS. Correlation of microRNA levels during hypoxia with predicted target mRNAs through genome-wide microarray analysis. BMC Med Genet. 2009;2:15.

5. Ramachandran S, Karp PH, Jiang P, Ostedgaard LS, Walz AE, Fisher JT, Keshavjee S, Lennox KA, Jacobi AM, Rose SD, Behlke MA, Welsh MJ, Xing Y, Mccray PB Jr. A microRNA network regulates expression and biosynthesis of wild-type and DeltaF508 mutant cystic fibrosis transmembrane conductance regulator. Proc Natl Acad Sci U S A. 2012;109:13362-7.

6. Singh SK, Pal Bhadra M, Girschick HJ, Bhadra U. MicroRNAs-micro in size but macro in function. FEBS J. 2008:275:4929-44.

7. Bartoszewska S, Kochan K, Madanecki P, Piotrowski A, Ochocka R, Collawn JF, Bartoszewski R. Regulation of the unfolded protein response by microRNAs. Cell Mol Biol Lett. 2013;18:555-78.

8. Lytle JR, Yario TA, Steitz JA. Target mRNAs are repressed as efficiently by microRNA-binding sites in the $5^{\prime}$ UTR as in the 3' UTR. Proc Natl Acad Sci U S A. 2007;104:9667-72.

9. Moszynska A, Gebert M, Collawn JF, Bartoszewski R. SNPs in microRNA target sites and their potential role in human disease. Open Biol. 2017;7:170019.

10. Madanecki P, Kapoor N, Bebok Z, Ochocka R, Collawn JF, Bartoszewski R. Regulation of angiogenesis by hypoxia: the role of microRNA. Cell Mol Biol Lett. 2013;18:47-57.

11. Bebok Z, Collawn JF, Wakefield J, Parker W, Li Y, Varga K, Sorscher EJ, Clancy JP. Failure of CAMP agonists to activate rescued deltaF508 CFTR in CFBE41o- airway epithelial monolayers. J Physiol. 2005;569:601-15.

12. Cozens AL, Yezzi MJ, Kunzelmann K, Ohrui T, Chin L, Eng K, Finkbeiner WE, Widdicombe JH, Gruenert DC. CFTR expression and chloride secretion in polarized immortal human bronchial epithelial cells. Am J Respir Cell Mol Biol. 1994;10:38-47.

13. Bartoszewski R, Rab A, Twitty G, Stevenson L, Fortenberry J, Piotrowski A, Dumanski JP, Bebok Z. The mechanism of cystic fibrosis transmembrane conductance regulator transcriptional repression during the unfolded protein response. J Biol Chem. 2008;283:12154-65.

14. Bartoszewski R, Rab A, Fu L, Bartoszewska S, Collawn J, Bebok Z. CFTR expression regulation by the unfolded protein response. Methods Enzymol. 2011:491:3-24.

15. Bartoszewski R, Hering A, Marszall M, Stefanowicz Hajduk J, Bartoszewska S, Kapoor N, Kochan K, Ochocka R. Mangiferin has an additive effect on the apoptotic properties of hesperidin in Cyclopia sp. tea extracts. PLoS One. 2014;9:e92128.

16. Larionov A, Krause A, Miller W. A standard curve based method for relative real time PCR data processing. BMC Bioinformatics. 2005;6:62.

17. Bartoszewski R, Kroliczewski J, Piotrowski A, Jasiecka AJ, Bartoszewska S, Vecchio-Pagan B, Fu L, Sobolewska A, Matalon S, Cutting GR, Rowe SM, Collawn JF. Codon bias and the folding dynamics of the cystic fibrosis transmembrane conductance regulator. Cell Mol Biol Lett. 2016;21:23.

18. Zheng W, Kuhlicke J, Jäckel K, Eltzschig HK, Singh A, Sjöblom M, Riederer B, Weinhold C, Seidler U, Colgan SP, Karhausen J. Hypoxia inducible factor-1 (HIF-1)-mediated repression of cystic fibrosis transmembrane conductance regulator (CFTR) in the intestinal epithelium. FASEB J. 2009;23:204-13.

19. Bebok Z, Varga K, Hicks JK, Venglarik CJ, Kovacs T, Chen L, Hardiman KM, Collawn JF, Sorscher EJ, Matalon S. Reactive oxygen nitrogen species decrease cystic fibrosis transmembrane conductance regulator expression and cAMP-mediated Cl- secretion in airway epithelia. J Biol Chem. 2002;277:43041-9.

20. Janaszak-Jasiecka A, Bartoszewska S, Kochan K, Piotrowski A, Kalinowski L, Kamysz W, Ochocka RJ, Bartoszewski R, Collawn JF. miR-429 regulates the transition between Hypoxia-Inducible Factor (HIF)1A and HIF3A expression in human endothelial cells. Sci Rep. 2016;6:22775.

21. Greco S, Martelli F. MicroRNAs in hypoxia response. Antioxid Redox Signal. 2014;21:1164-6.

22. Betel D, Koppal A, Agius P, Sander C, Leslie C. Comprehensive modeling of microRNA targets predicts functional non-conserved and non-canonical sites. Genome Biol. 2010;11:R90.

23. Agarwal V, Bell GW, Nam JW, Bartel DP. Predicting effective microRNA target sites in mammalian mRNAs. elife. 2015:4:e05005.

24. Bartoszewska S, Kochan K, Piotrowski A, Kamysz W, Ochocka RJ, Collawn JF, Bartoszewski R. The hypoxia-inducible miR-429 regulates hypoxia-inducible factor-1alpha expression in human endothelial cells through a negative feedback loop. FASEB J. 2015;29:1467-79.

25. McKiernan PJ, Greene CM. MicroRNA dysregulation in cystic fibrosis. Mediators Inflamm. 2015;2015:529642.

26. Sonneville F, Ruffin M, Guillot L, Rousselet N, Le Rouzic P, Corvol H, Tabary O. New insights about miRNAs in cystic fibrosis. Am J Pathol. 2015;185(4):897-908.

27. Noel $\mathrm{S}$, Leal T. Emerging roles of microRNAs in cystic fibrosis — from pathogenesis to development of new therapies. In: Wat D, editor. Cystic fibrosis in the light of new research. Rijeka: InTech; 2015. p. Ch. 10.

28. Booton R, Lindsay MA. Emerging role of MicroRNAs and long noncoding RNAs in respiratory disease. Chest. 2014; 146:193-204.

29. Oglesby IK, Chotirmall SH, Mcelvaney NG, Greene CM. Regulation of cystic fibrosis transmembrane conductance regulator by microRNA-145, -223 , and -494 is altered in DeltaF508 cystic fibrosis airway epithelium. J Immunol. 2013;190:3354-62.

30. Amato F, Seia M, Giordano S, Elce A, Zarrilli F, Castaldo G, Tomaiuolo R. Gene mutation in MicroRNA target sites of CFTR gene: a novel pathogenetic mechanism in cystic fibrosis? PLoS One. 2013;8:e60448. 